\title{
Changes of Platelet Indices in Juvenile Idiopathic Arthritis in Acute Phase and After Two Months Treatment
}

\author{
Marjan Vakili,, Vahid Ziaee, ${ }^{2,3,}{ }^{,}$Mohammad Hassan Moradinejad, ${ }^{1,3}$ Seyed Reza Raeeskarami, ${ }^{3}$ Farzad \\ Kompani, ${ }^{1,3}$ and Tayebeh Rahamooz ${ }^{1}$ \\ ${ }^{1}$ Children's Medical Center, Pediatrics Center of Excellence, Tehran University of Medical Sciences, Tehran, IR Iran \\ ${ }^{2}$ Rheumatology Research Center, Tehran University of Medical Sciences, Tehran, IR Iran \\ ${ }^{3}$ Department of Pediatrics, Tehran University of Medical Sciences, Tehran, IR Iran \\ Corresponding author: Vahid Ziaee, Department of Pediatrics, Tehran University of Medical Sciences, Tehran, IR Iran. E-mail: ziaee@tums.ac.ir
}

Received 2015 December 09; Revised 2015 December 25; Accepted 2016 January 05.

\begin{abstract}
Background: Various indices have been raised as predictors of activity and severity of juvenile idiopathic arthritis.

Objectives: This study was conducted to investigate the changes of platelet indices in acute phase and two months after treatment in these patients. Patients and Methods: In a cohort study, platelet count, mean platelet volume (MPV), platelet distribution width (PDW), plateletcrit (PCT) were evaluated in children referred to children's medical center, Tehran due to juvenile idiopathic arthritis from March 2013 to March 2014 during the acute phase and two months after standard treatment. The statistical data were analyzed by SPSS 19 software, and the significance level was set as P $<0.05$.

Results: In this study, 55 children (24 boys and 31 girls) with mean \pm SD age of $7.50 \pm 3.35$ years were studied. The mean \pm SD value of platelet count was $441872.7 \pm 151836.9$ in the acute phase and reached $395418.2 \pm 119601.6$ two months after treatment $(P=0.01)$. The mean \pm SD PCT in the acute phase of various subtypes of the disease was $0.32 \pm 0.11$, which reached $0.29 \pm 0.10$ after treatment $(P=0.09)$. However, the PDW range in different subtypes of the disease reached $13.4 \pm 8.0$ from $13.9 \pm 2.9$ and MPV reached $8.7 \pm 0.9$ from $8.8 \pm 1.1$ after treatment, but they were not significantly different from the results in the acute phase $(\mathrm{P}=0.5)$.

Conclusions: Platelet count is one of the most remarkable indices in JIA. Evaluation of PCT can also help determine the severity of the inflammatory process in the follow-up and treatment process.
\end{abstract}

Keywords: Juvenile Idiopathic Arthritis, Acute Phase Reactants, Platelet Count, Platelet Indices, Mean Platelet Volume

\section{Background}

As the most common type of rheumatologic disease during childhood, juvenile idiopathic arthritis (JIA) is in fact a set of arthritis conditions before the age of 16 with unknown etiology that lasts longer than 6 weeks $(1,2)$. Although the etiology and pathogenesis of JIA is unknown; it seems that various types of HLA class I and II, polymorphism in genes related to Tumor necrosis factors- $\alpha$ (TNF- $\alpha$ ), interlukines IL-6, and IL-1 secreted from monocyte cells and macrophages, some environmental factors and trauma to joints contribute to emergence of the disease(3). However, autoimmunity has a main role in the progression of this disease. In fact, T cells release proinflammatory cytokines such as L-6, TNF- $\alpha$ and IL-1, and thus cause hypertrophy and hyperplasia of villous with hyperemia and synovial tissue edema (4).

One of the effects of proinflammatory cytokines produced in response to systemic inflammation in the liver is megakaryocyte stimulation and thus increases in the number of platelets in response to inflammation $(3,5)$. This results in the production of larger and more reactive platelets (6). There is evidence that platelets penetrate to synovial fluid in rheumatoid arthritis (RA) (7, 8). These platelets can bind to endothelial cells and leukocytes in inflamed synovial blood vessels causing thrombosis and abnormalities in synovial perfusion and joint destruction (9). Therefore, platelets may also contribute to the pathogenesis of JIA.

Few studies have been conducted on changes in platelet indices in adult rheumatoid arthritis. Mean platelet volume (MPV) is one of the markers that can be suggestive of platelet response. In physiological terms, increased MPV may suggest the entry of greater number of young platelets due to their higher production in blood. Some studies suggest MPV changes in the active phase of rheumatoid arthritis. In a few studies, MPV was used for monitoring response to treatment in rheumatoid arthritis $(10,11)$. In a study, treatment with anti-TNFa was associated with a significant increase in MPV (10). Another study was conducted on the relationship between MPV and clinical activity indices of RA and ankylosing spondylitis and the results showed that MPV was lower in the active phase of RA and AS compared to the control group and was significantly increased in the patient group after treatment (12). However, a study on JIA patients showed increased MPV in 
active phase of JIA (11).

\section{Objectives}

Given that no study has been conducted on the changes in the inflammatory phase reactants in patients with a variety of JIA in the course of the disease, we investigated the change pattern of platelet indices in the acute phase and after disease control.

\section{Patients and Methods}

This cohort study evaluated children whose JIA was diagnosed according to international league of associations for rheumatology (ILAR) from March 2013 to March 2014 (13). All patients were selected from pediatric rheumatology division in a tertiary children's medical center in Tehran, Iran. JIA patients, systemic type and pauci-and polyarticular form (with RF negative) enrolled in this study. Other types include RF positive ployarticular form, psoriasis arthritis; enthesitis related arthritis and undifferentiated arthritis were not enrolled in this study due to low sample size in each group. Patients were excluded from the study if they failed to take part in follow-up examinations, the diagnosis of JIA changed during the first month or they simultaneously had the symptoms of infectious diseases. In addition to treatment with methotrexate, all patients were treated with prednisolone or nonsteroidal anti-inflammatory drugs (NSAIDs), based on the severity of symptoms and disease subtype.

According to a similar previous RA study (10), sample size was calculated to be 20 cases. Because we decided to study subtypes of JIA, we considered 60 cases for this study. These patients were studied two months after initiating the treatment and remission of the overt symptoms of the disease in terms of platelet indices including platelet count, MPV, plateletcrit (PCT) and platelet distribution width (PDW). Mindray® automated counter was used to evaluate platelet indices. Normal values of platelet factors were considered based on the following (14):

Platelet (PLT) count range: $156-373 \times 10^{9} / \mathrm{L}$,

MPV range: 7.4 - $10.4 \mathrm{fL}$,

PDW range: 15.6 - $18.2 \mathrm{fL}$,

PCT range: $0.155 \%-0.320 \%$.

This study was conducted after approval by the ethics committee of the Tehran University of Medical Sciences. All data were gathered from patients' records. Verbal consents were obtained from parents before data collection. The data were analyzed by SPSS 19 software, qualitative data were compared by Chi-square test and quantitative data by $t$ test between the two groups; the Dunn-Bonferroni post hoc test, Wilcoxon or Kruskal-Wallis were used to compare the means; quantitative data were reported as mean \pm standard deviation $\pm S D$ and qualitative data as frequency. In all cases, the significance level was set as $\mathrm{P}<0.05$.

\section{Results}

In this study, a total of 55 patients diagnosed with JIA were examined of whom 24 (43.6\%) were boys and 31 (54.4\%) girls. Patients' mean \pm SD age was $7.50 \pm 3.35$ years (from 1 to 14 years). In terms of disease subtype, 27 (49.1\%) patients had oligoarthritis, 19 patients (34.5\%) polyarthritis and 9 (16.4\%) had systemic arthritis.

Table 1 shows mean \pm SD values of platelet count and platelet indices in patients. The difference between platelet count and PCT was significant in all subtypes of the disease in the acute phase $(\mathrm{P}=0.01$ and $\mathrm{P}=0.02$, respectively). In analysis among the subtypes of JIA, mean difference of MPV and PCT had significant differences between oligoarticular and polyarticular subtype in the acute phase (both $\mathrm{P}=0.03$ ). On the other hand significant difference in the acute phase was related to differences between oligoarticular and polyarticular subtype.

After 2 months treatment, there was still a statistically significant difference between the mean platelet count in all three subtypes of the disease $(\mathrm{P}=0.03)$. There was no significant difference between the mean platelet indices in different subtypes of the disease although this difference was significant in PCT as borderline $(\mathrm{P}=0.09)$ (Table 2).

Table 3 presents the comparison of the number of platelets and platelet indices before and two months after treatment. As shown, the total number of platelets and also the number of platelets in the systemic disease declined after treatment $(\mathrm{P}=01, \mathrm{P}<0.01$, respectively). Also systemic PCT significantly reduced after treatment (P $=0.02$ ) and the total value of PCT indicated a significant borderline reduction after treatment $(\mathrm{P}=0.09)$. The index showed no significant change in other subtypes of the disease. Also, other platelet indices i.e. PDW and MPV had no significant changes overall and in subtypes of the disease $(\mathrm{P}=0.5$ for each one).

\section{Discussion}

Clinical presentations of JIA are highly variable such that it can be seen in a range of very mild self-limiting arthritis to highly destructive arthritis. However, no measure has been developed so far to evaluate the response rate to treatment. Also, no specific and sensitive marker has been identified to assess disease activity rate (15). Evaluation of acute phase reactants such as platelet indices is 
Table 1. Comparison of Indices in Patients With Different Subtypes of JIA in Acute Phase of Disease

\begin{tabular}{|c|c|c|c|}
\hline Parameter & \multirow[t]{2}{*}{ Mean \pm SD } & \multicolumn{2}{|c|}{ 95\% Confidence Interval } \\
\hline & & Lower Bound & Upper Bound \\
\hline \multicolumn{4}{|l|}{ PLT $^{\mathrm{a}}$} \\
\hline Oligo-articular, $n=27$ & $380629.6 \pm 137244.9$ & 326337.35 & 434921.91 \\
\hline Poly-articular, n = 19 & $481842.1 \pm 122358.0$ & 422867.45 & 540816.76 \\
\hline Total, $\mathrm{n}=55$ & $441872.7 \pm 151836.9$ & 400825.46 & 482919.99 \\
\hline \multicolumn{4}{|l|}{ PDW } \\
\hline Oligo-articular, n= 27 & $14.2 \pm 2.9$ & 13.0 & 15.3 \\
\hline Poly-articular, n = 19 & $13.8 \pm 3.0$ & 12.4 & 15.2 \\
\hline Systemic, $\mathrm{n}=9$ & $13.5 \pm 3.1$ & 11.1 & 16.0 \\
\hline Total, $\mathrm{n}=55$ & $13.9 \pm 2.9$ & 13.2 & 14.7 \\
\hline \multicolumn{4}{|l|}{$\mathbf{P C T}^{\mathbf{a}}$} \\
\hline Oligo-articular, n= 27 & $0.28 \pm 0.10$ & 0.24 & 0.32 \\
\hline Poly-articular, n = 19 & $0.35 \pm 0.10$ & 0.31 & 0.40 \\
\hline Systemic, $\mathrm{n}=9$ & $0.36 \pm 0.11$ & 0.27 & 0.44 \\
\hline Total, $\mathrm{n}=55$ & $0.32 \pm 0.11$ & 0.29 & 0.35 \\
\hline \multicolumn{4}{|l|}{ MPV $^{\mathbf{a}}$} \\
\hline Oligo-articular, n= 27 & $9.2 \pm 1.1$ & 8.8 & 9.7 \\
\hline Poly-articular, n = 19 & $8.4 \pm 0.9$ & 8.0 & 8.8 \\
\hline Systemic, $\mathrm{n}=9$ & $8.5 \pm 0.9$ & 7.8 & 9.2 \\
\hline Total, $\mathrm{n}=55$ & $8.8 \pm 1.1$ & 8.5 & 9.1 \\
\hline
\end{tabular}

Abbreviations: SD, standard deviation; JIA, juvenile idiopathic arthritis; PLT, platelet; PDW, platelet distribution width; MPV, Mean platelet volume; PCT, plateletcrit. ${ }^{\mathrm{a}}$ Significant $(\mathrm{P}<0.05)$.

a way to evaluate these patients (3). Although few studies have been conducted on platelet indices in rheumatologic diseases, studies on these factors in JIA are much fewer.

In this study, increased mean platelet count in the acute phase of the disease was seen in the systemic and polyarticular subtypes; however, mean platelet count was normal in oligoarticular subtype. Interleukin 6 (IL-6) is the cause of thrombocytosis in autoimmune diseases such as RA (16). As reactive thrombocytosis is a symptom of inflammation in JIA $(17,18)$, this represents a more severe inflammatory process in the polyarticular and systemic subtypes. In other studies, inflammatory process has been reported in the systemic subtype of JIA $(19,20)$. Spiegel (17) also found that thrombocytosis in an active systemic disease can be considered as an important factor in the poor prognosis of the patients.

In this study, after 2 months of treatment, the mean platelet count was evaluated again in all three subtypes of the disease, which indicated a decrease in all of them.
The reduction in mean platelet count after treatment compared to before treatment was statistically significant. In polyarticular subtype, the mean platelet count was still above the normal range despite two months of treatment, although this variable had reached normal range in the systemic subtype like oligoarticular involvement. This can be a sign of slower remission of inflammatory symptoms in polyarticular subtype with mild treatments compared to systemic disease. Ozturk et al. (14) showed that the mean number of platelets is reduced in Crohn's and ulcerative colitis remission phase compared to the active phase. Berntson et al. (2) reported that the mean number of platelets in patients with JIA with at least one joint involvement is significantly higher than the mean platelet count in patients who are in complete remission. However, the number of platelets higher than the normal range was not necessarily associated with a symptomatic disease.

In another study conducted by Sandborg et al. (21), predicting measures of joint destruction were evaluated in 
Table 2. Comparison of Indices in Patients With Different Subtypes of JIA After 2 Months Treatment

\begin{tabular}{|c|c|c|c|}
\hline Parameter & \multirow[t]{2}{*}{ Mean \pm SD } & \multicolumn{2}{|c|}{ 95\% Confidence Interval } \\
\hline & & Lower Bound & Upper Bound \\
\hline \multicolumn{4}{|l|}{$\mathbf{P L T}^{\mathrm{a}}$} \\
\hline Oligo-articular, n= 27 & $358444.4 \pm 91415.1$ & 322281.8 & 394607.1 \\
\hline Poly-articular, n = 19 & $453105.3 \pm 134702.1$ & 388180.9 & 518029.6 \\
\hline Total, $\mathrm{n}=55$ & $395418.2 \pm 119601.6$ & 363085.3 & 427751.0 \\
\hline \multicolumn{4}{|l|}{ PDW } \\
\hline Oligo-articular, n= 27 & $13.1 \pm 5.7$ & 10.8 & 15.3310 \\
\hline Poly-articular, $\mathrm{n}=19$ & $12.7 \pm 8.8$ & 8.5 & 17.0 \\
\hline Systemic, $\mathrm{n}=9$ & $16.2 \pm 11.9$ & 7.0 & 25.3 \\
\hline Total, $\mathrm{n}=55$ & $13.4 \pm 8.0$ & 11.2 & 15.6 \\
\hline \multicolumn{4}{|l|}{ PCT } \\
\hline Oligo-articular, n= 27 & $0.27 \pm 0.09$ & 0.24 & 0.31 \\
\hline Poly-articular, n=19 & $0.33 \pm 0.12$ & 0.28 & 0.39 \\
\hline Systemic, $\mathrm{n}=9$ & $0.26 \pm 0.10$ & 0.18 & 0.33 \\
\hline Total, $\mathrm{n}=55$ & $0.29 \pm 0.10$ & 0.26 & 0.32 \\
\hline \multicolumn{4}{|l|}{ MPV } \\
\hline Oligo-articular, n = 27 & $8.9 \pm 1.0$ & 8.5 & 9.3 \\
\hline Poly-articular, n = 19 & $8.6 \pm 0.67$ & 8.3 & 8.9 \\
\hline Systemic, $\mathrm{n}=9$ & $8.6 \pm 0.8$ & 7.9 & 9.2 \\
\hline Total, $\mathrm{n}=55$ & $8.7 \pm 0.9$ & 8.5 & 9.0 \\
\hline
\end{tabular}

Abbreviations: SD, standard deviation; JIA, juvenile idiopathic arthritis; PLT, platelet; PDW, platelet distribution width; MPV, Mean platelet volume; PCT, plateletcrit. ${ }^{\mathrm{a}}$ Significant $(\mathrm{P}<0.05)$.

JIA. This study showed that children who had more than 788,000 platelets at least once in the first 3 months, and also children with more than 13 joints involved, were more likely to develop joint destruction within 2 years, despite platelet count less than 788,000. Spiegel et al. (17) also showed that with each 100,000 increase in platelet count in the tests performed 6 months after diagnosis, the risk of severe disease with adverse outcomes in patients with systemic JIA would be 1.45 times greater. Milovanovic et al. (5) found that the mean number of platelets in the active phase of rheumatoid arthritis is significantly higher than in remission phase of the disease, and the increase in the number of platelets is associated with IL-6, but not with thrombopoietin.

MPV is a platelet index that is measured automatically, is associated with platelets function and activity $(22,23)$, and is affected by inflammatory reactions. Inflammatory mediators stimulate bone marrow precursors in the presence of autoimmune diseases to produce more platelets through shortening their maturation time, so smaller platelets enter the blood stream, while active platelets are destroyed at the site of inflammation (24). However, there is a nonlinear inverse relationship between MPV and platelet count (25).

In this study, MPV was within normal range in the acute phase in all cases. The highest MPV was observed in oligoarticular subtype, and then in the systemic and polyarticular subtype, however, MPV difference was not significant between systemic and polyarticular form in the acute phase. It seems that MPV has an inverse relationship with inflammatory disease severity such that studies by Isik et al. (26) and Gasparyan et al. (27) suggest a decline in MPV in severe inflammatory diseases such as rheumatoid arthritis active phase. In most cases; however, it should be considered that MPV is in the low normal range (26). In contrast, in a study on JIA, MPV increased in acute phase and in correlation to severe disease (11). In our study, after 2 months of treatment, MPV was assessed again in all three disease subtypes, 
Table 3. Comparison of Indices in Acute Phase of Disease and 2 Months after treatment in Different Subtypes of JIA

\begin{tabular}{|c|c|c|c|}
\hline Parameter & \multirow[t]{2}{*}{ Mean \pm SD } & \multicolumn{2}{|c|}{ 95\% Confidence Interval } \\
\hline & & Lower Bound & Upper Bound \\
\hline \multicolumn{4}{|l|}{$\mathbf{P L T}^{\mathrm{a}}$} \\
\hline Oligo-articular, $n=27$ & $22185.2 \pm 20674.0$ & -20310.8 & 64681.1 \\
\hline Poly-articular, n = 19 & $28736.8 \pm 28179.7$ & -30466.6 & 87940.3 \\
\hline Systemic, n = 9 & $156666.7 \pm 36913.2^{*}$ & 71544.7 & 241788.6 \\
\hline Total, $\mathrm{n}=55$ & $46454.5^{*}$ & 13531.161 & 79377.930 \\
\hline \multicolumn{4}{|l|}{ PDW } \\
\hline Oligo-articular, $n=27$ & $1.1 \pm 1.2$ & -1.25 & 3.5 \\
\hline Poly-articular, n = 19 & $1.1 \pm 1.9$ & -3.1 & 5.2 \\
\hline Systemic, $\mathrm{n}=9$ & $-2.6 \pm 4.3$ & -12.7 & 7.5 \\
\hline Total, $\mathrm{n}=55$ & 0.49 & -1.77 & 2.76 \\
\hline \multicolumn{4}{|l|}{ PCT } \\
\hline Oligo-articular, $\mathrm{n}=27$ & $0.004 \pm 0.021$ & -0.039 & 0.047 \\
\hline Poly-articular, n = 19 & $0.023 \pm 0.026$ & -0.032 & 0.079 \\
\hline Systemic, $\mathrm{n}=9$ & $0.100 \pm 0.033$ & 0.023 & 0.177 \\
\hline Total, $\mathrm{n}=55$ & 0.03 & 0.00 & 0.06 \\
\hline \multicolumn{4}{|l|}{ MPV } \\
\hline Oligo-articular, $n=27$ & $0.33 \pm 0.21$ & -0.10 & 0.76 \\
\hline Poly-articular, n = 19 & $-0.19 \pm 0.16$ & -0.53 & 0.15 \\
\hline Systemic, $\mathrm{n}=9$ & $0.00 \pm 0.43$ & -0.99 & 0.99 \\
\hline Total, $\mathrm{n}=55$ & 0.10 & -0.18 & 0.37 \\
\hline
\end{tabular}

Abbreviations: SD, standard deviation; JIA, juvenile idiopathic arthritis; PLT, platelet; PDW, platelet distribution width; MPV, Mean platelet volume; PCT, plateletcrit. ${ }^{\mathrm{a}}$ Significant $(\mathrm{P}<0.05)$.

and despite a modest rise in the polyarticular subtype, it had a decrease in other two subtypes. However, differences were not statistically significant after treatment compared to MPV before treatment and the rate was within normal limits in all cases. Studies by Isık (26) and Gasparyan (27) also showed that MPV that decreased in the active phase of rheumatoid arthritis as a severe inflammatory disease would increase after remission to a normal range [7.4 - 10.4 $\mathrm{fL}$. Gasparyan et al. (27) depicted that MPV is decreased in severe inflammatory diseases, but is increased in mild inflammatory diseases. So it seems that increased MPV in subsystemic type after two months of treatment can represent the appropriate effect of treatment in the control of inflammatory response in these patients. Studies of Kapsoritakis et al. (28) and Yuksel et al. (29) also showed that there is an inverse relationship between the severity of inflammatory bowel disease in patients with IBD and MPV. However, Ozturk et al. (14) found that MPV was decreased after the remission of acute phase of ulcerative colitis and increased in remission of Crohn's disease. So it seems that the evaluation of MPV changes alone is not helpful in the follow-up and evaluation of inflammatory diseases because MPV showed a modest rise in polyarticular subtype and a decrease in other subtypes of the disease two months after treatment in our study. However, in most cases, MPV was in the low normal range in the acute phase and two months after treatment. Milovanovic et al. (5) also believe that because of the overlap of MPV in inflammatory diseases with normal values, it is necessary that changes of this index be individually evaluated and interpreted in the course of the assessment of each patient.

PDW is another indicator of platelet volume directly measured by flow cytometry, which reports platelet distribution by evaluating the top $20 \%$ of the distribution curve (30). This study showed that PDW was reduced in all subtypes in the acute phase of the disease, and the highest drop in PDW was observed in systemic subtype, then in polyarticular and oligoarticular subtype. According to the 
results of Isık et al. (26), it seems that PDW like MPV has an inverse relationship with inflammatory disease severity and is reduced in severe inflammatory diseases such as the active phase in rheumatoid arthritis. Ozturk et al. (14) also found that PDW has an inverse relationship with ulcerative colitis activity. In our study, after 2 months of treatment, the mean PDW was evaluated again in all three subtypes of the disease, and despite an increase in the systemic subtype, it showed a decrease in other two subtypes, but PDW changes were not statistically significant before and after treatment. The index only reached a low normal range in systemic subtype and was still lower than the normal range in other two subtypes (15.6 - 18.2 fL). Isık et al. (26) also showed that PDW is decreased in the active phase of rheumatoid arthritis as a severe inflammatory disease; however, unlike our study, the mean PDW in the study of Isık was in low normal range. Ozturk et al. (14) reported that PDW which is decreased in the active phase of inflammatory bowel diseases is increased after the remission of acute phase of Crohn's disease and ulcerative colitis and can be used as a good indicator to track inflammatory bowel diseases from acute phase to remission. In contrast to previous studies on JIA patients, Gunes et al. (11) reported, similar to MPV, higher range for PDW in acute phase and in patients with severe disease. However, in the present study, the evaluation of PDW changes was not helpful in follow-up and disease assessment.

Unlike other platelet-related indices that are measured directly by a machine, PCT is a measure that depends on platelet count and MPV, and shows platelets in a unit of blood volume (31). This study showed that an increase in the mean PCT in the acute phase of the disease is observed in systemic and polyarticular subtypes; however, mean PCT in oligoarticular subtype was within the normal range. Studies of Isık et al. (26) and Santimone et al. (32) suggest an increase in PCT in severe inflammatory diseases such as rheumatoid arthritis active phase; however, it should be considered that despite the increase mentioned, PCT is in high normal range in most cases (26). The study of Ozturk et al. (14) also found that PCT is increased with ulcerative colitis activity. In our study, the mean PCT was evaluated again after 2 months of treatment in all three subtypes of the disease, and despite the lack of significant changes in the oligoarticular subtype, it was decreased in two other subtypes. These changes after treatment were only statistically significant in systemic subtype, compared to the mean PCT before treatment. This index reached a normal range only in the systemic subtype after treatment and was still higher than the normal range in the polyarticular subtype; this can be a sign of slower remission of inflammation symptoms in treatment of polyarticular disease as compared to the systemic subtype. Ozturk et al. (14) showed that PCT which is increased in the active phase of inflammatory bowel diseases can be used as a good indicator to track IBD from the acute phase to remission. The study of Isık et al. (26) also indicated that PCT is higher in the active phase of rheumatoid arthritis than remission period of the disease. According to the results of our study it seems that the evaluation of PCT changes is only helpful in the follow up and assessment of the disease in the systemic and polyarticular subtypes as in our study this measure was within normal limits even before the treatment in oligoarticular subtype.

Confounding factors and severity of the JIA has not been considered in this study. In a recent study, platelets indices were compared with severity of the rheumatoid arthritis and JIA $(11,33)$. Other studies are recommended for covering these limitations and considering severity in subtype of JIA patients.

\subsection{Conclusion}

This study showed that JIA leads to some changes in platelet counts and platelet indices, especially in the acute phase of the disease in different subtypes with developing a chronic inflammatory process. On the other hand, although in most cases only platelet count is considered as an indicator of acute inflammation, PCT evaluation can also be helpful in determining the severity of the inflammatory process in the course of follow up and treatment. However, MPV and PDW had no significant changes after treatment. Since platelet indices are non-specific for autoimmune diseases, these findings can be considered and interpreted besides other clinical and laboratory findings.

\section{Acknowledgments}

This study is a part of thesis by Dr. M. Vakili, approved by research committee of faculty of Medicine, Tehran University of Medical Sciences.

\section{Footnote}

Authors' Contribution: Marjan Vakili participated in the acquisition of data and manuscript preparation; Vahid Ziaee participated in the concept and Design carried of the study, data collection, analysis and interpretation and critical revision of the manuscript; Mohammad Hassan Moradinejad participated in the concept and design carried of the study and critical revision of the manuscript; Seyed Reza Raeeskarami and Farzad Kompani participated in the design of the study and interpretation of findings; Tayebeh Rahamooz participated in the acquisition of data. All authors read and approved the final manuscript. 


\section{References}

1. Borchers AT, Selmi C, Cheema G, Keen CL, Shoenfeld Y, Gershwin ME. Juvenile idiopathic arthritis. Autoimmun Rev. 2006;5(4):279-98. doi: 10.1016/j.autrev.2005.09.011. [PubMed:16697970].

2. Berntson L, Wernroth L, Fasth A, Aalto K, Herlin T, Nielsen S, et al. Assessment of disease activity in juvenile idiopathic arthritis. The number and the size of joints matter. J Rheumatol. 2007;34(10):2106-11. [PubMed: 17896798].

3. Breda L, Nozzi M, De Sanctis S, Chiarelli F. Laboratory tests in the diagnosis and follow-up of pediatric rheumatic diseases: an update. Semin Arthritis Rheum. 2010;40(1):53-72. doi: 10.1016/j.semarthrit.2008.12.001. [PubMed: 19246077].

4. Alamanos Y, Voulgari PV, Drosos AA. Incidence and prevalence of rheumatoid arthritis, based on the 1987 American College of Rheumatology criteria: a systematic review. Semin Arthritis Rheum. 2006;36(3):182-8. doi: 10.1016/j.semarthrit.2006.08.006. [PubMed: 17045630].

5. Milovanovic M, Nilsson E, Jaremo P. Relationships between platelets and inflammatory markers in rheumatoid arthritis. Clin Chim Acta. 2004;343(1-2):237-40. doi: 10.1016/j.cccn.2003.12.030. [PubMed: 15115702].

6. Jagroop IA, Clatworthy I, Lewin J, Mikhailidis DP. Shape change in human platelets: measurement with a channelyzer and visualisation by electron microscopy. Platelets. 2000;11(1):28-32. [PubMed: 10938878].

7. Schmitt-Sody M, Klose A, Gottschalk O, Metz P, Gebhard H, Zysk $S$, et al. Platelet-endothelial cell interactions in murine antigeninduced arthritis. Rheumatology (Oxford). 2005;44(7):885-9. doi: 10.1093/rheumatology/keh638. [PubMed: 15827038].

8. Gasparyan AY, Stavropoulos-Kalinoglou A, Mikhailidis DP, Douglas KM, Kitas GD. Platelet function in rheumatoid arthritis: arthritic and cardiovascular implications. Rheumatol Int. 2011;31(2):153-64. doi: 10.1007/s00296-010-1446-x. [PubMed: 20390282].

9. Schmitt-Sody M, Metz P, Gottschalk O, Birkenmaier C, Zysk S, Veihelmann A, et al. Platelet P-selectin is significantly involved in leukocyteendothelial cell interaction in murine antigen-induced arthritis. Platelets. 2007;18(5):365-72. doi:10.1080/09537100701191315. [PubMed: 17654306].

10. Gasparyan AY, Sandoo A, Stavropoulos-Kalinoglou A, Kitas GD. Mean platelet volume in patients with rheumatoid arthritis: the effect of anti-TNF-alpha therapy. Rheumatol Int. 2010;30(8):1125-9. doi: 10.1007/s00296-009-1345-1. [PubMed: 20066426].

11. Gunes A, Ece A, Sen V, Uluca U, Aktar F, Tan I, et al. Correlation of mean platelet volume, neutrophil-to-lymphocyte ratio, and disease activity in children with juvenile idiopathic arthritis. Int J Clin Exp Med. 2015;8(7):11337-41. [PubMed: 26379946].

12. Kisacik B, Tufan A, Kalyoncu U, Karadag O, Akdogan A, Ozturk MA, et al. Mean platelet volume (MPV) as an inflammatory marker in ankylosing spondylitis and rheumatoid arthritis. Joint Bone Spine. 2008;75(3):291-4. doi: 10.1016/j.jbspin.2007.06.016. [PubMed: 18403245].

13. Petty RE, Southwood TR, Manners P, Baum J, Glass DN, Goldenberg J, et al. International League of Associations for Rheumatology classification of juvenile idiopathic arthritis: second revision, Edmonton, 2001. J Rheumatol. 2004;31(2):390-2. [PubMed:14760812].

14. Ozturk ZA, Dag MS, Kuyumcu ME, Cam H, Yesil Y, Yilmaz N, et al. Could platelet indices be new biomarkers for inflammatory bowel diseases?. Eur Rev Med Pharmacol Sci. 2013;17(3):334-41. [PubMed: 23426536].

15. Lurati A, Pontikaki I, Teruzzi B, Desiati F, Gerloni V, Gattinara M, et al. A comparison of response criteria to evaluate therapeutic response in patients with juvenile idiopathic arthritis treated with methotrexate and/or anti-tumor necrosis factor alpha agents. Arthritis Rheum. 2006;54(5):1602-7. doi: 10.1002/art.21784. [PubMed: 16646003].

16. Ertenli I, Kiraz S, Ozturk MA, Haznedaroglu I, Celik I, Calguneri M. Pathologic thrombopoiesis of rheumatoid arthritis. Rheumatol
Int. 2003;23(2):49-60. doi: 10.1007/s00296-003-0289-0. [PubMed: 12634936].

17. Spiegel LR, Schneider R, Lang BA, Birdi N, Silverman ED, Laxer RM, et al. Early predictors of poor functional outcome in systemic-onset juvenile rheumatoid arthritis: a multicenter cohort study. Arthritis Rheum. 2000;43(11):2402-9.

18. Khaleel KJ, Ahmed AA, Alwash MM, Yasin NY. Mean platelet volume, platelet distribution width and plateletcrit values in differentiating clonal from secondary thrombocytosis. Med J Babylon. 2013;10(2):4738

19. Ravelli A, Martini A. Juvenile idiopathic arthritis. Lancet. 2007;369(9563):767-78. doi:10.1016/S0140-6736(07)60363-8. [PubMed: 17336654].

20. Prakken B, Albani S, Martini A. Juvenile idiopathic arthritis. Lancet. 2011;377(9783):2138-49. doi: 10.1016/S0140-6736(11)60244-4. [PubMed: 21684384].

21. Sandborg C, Holmes TH, Lee T, Biederman K, Bloch DA, Emery H, et al. Candidate early predictors for progression to joint damage in systemic juvenile idiopathic arthritis. J Rheumatol. 2006;33(11):2322-9. [PubMed: 16960920].

22. Threatte GA. Usefulness of the mean platelet volume. Clin Lab Med. 1993;13(4):937-50. [PubMed: 8313690].

23. Bath PM, Butterworth RJ. Platelet size: measurement, physiology and vascular disease. Blood Coagul Fibrinolysis. 1996;7(2):157-61. [PubMed: 8735807].

24. Voudoukis E, Karmiris K, Koutroubakis IE. Multipotent role of platelets in inflammatory bowel diseases: a clinical approach. World J Gastroenterol. 2014;20(12):3180-90. doi: 10.3748/wjg.v20.i12.3180. [PubMed: 24696603].

25. Berger JS, Eraso LH, Xie D, Sha D, Mohler E3. Mean platelet volume and prevalence of peripheral artery disease, the National Health and Nutrition Examination Survey, 1999-2004. Atherosclerosis. 2010;213(2):586-91. doi: 10.1016/j.atherosclerosis.2010.09.010. [PubMed: 20940069].

26. Isık M. , Sahin H. , Huseyin E. . New platelet indices as inflammatory parameters for patients with rheumatoid arthritis. Eur J Rheumatol. 2014;4:144-6.

27. Gasparyan AY, Ayvazyan L, Mikhailidis DP, Kitas GD. Mean platelet volume: a link between thrombosis and inflammation?. Curr Pharm Des. 2011;17(1):47-58. [PubMed: 21247392].

28. Kapsoritakis AN, Koukourakis MI, Sfiridaki A, Potamianos SP, Kosmadaki MG, Koutroubakis IE, et al. Mean platelet volume: a useful marker of inflammatory bowel disease activity. Am J Gastroenterol. 2001;96(3):776-81. doi: 10.1111/j.1572-0241.2001.03621.x. [PubMed: $11280550]$.

29. Yuksel O, Helvaci K, Basar O, Koklu S, Caner S, Helvaci N, et al. An overlooked indicator of disease activity in ulcerative colitis: mean platelet volume. Platelets. 2009;20(4):277-81. doi: 10.1080/09537100902856781. [PubMed: 19459134].

30. Vagdatli E, Gounari E, Lazaridou E, Katsibourlia E, Tsikopoulou F, Labrianou I. Platelet distribution width: a simple, practical and specific marker of activation of coagulation. Hippokratia. 2010;14(1):2832. [PubMed: 20411056].

31. Briggs C, Bain BJ. Basic haematological techniques. 11 ed. USA: Churchill Livingstone; 2011. pp. 30-51.

32. Santimone I, Di Castelnuovo A, De Curtis A, Spinelli M, Cugino D, Gianfagna F, et al. White blood cell count, sex and age are major determinants of heterogeneity of platelet indices in an adult general population: results from the MOLI-SANI project. Haematologica. 2011;96(8):1180-8. doi: 10.3324/haematol.2011.043042. [PubMed: 21546503].

33. Fu H, Qin B, Hu Z, Ma N, Yang M, Wei T, et al. Neutrophil- and platelet-tolymphocyte ratios are correlated with disease activity in rheumatoid arthritis. Clin Lab. 2015;61(3-4):269-73. [PubMed: 25974992]. 